\title{
Existence of Solutions for Anti-Periodic Fractional Differential Inclusions with $\psi$-Caupto Fractional Derivative
}

\author{
Dandan Yang $(\mathbb{D}$ and Chuanzhi Bai \\ School of Mathematical Science, Huaiyin Normal University, Huaian, Jiangsu 223300, China \\ Correspondence should be addressed to Dandan Yang; ydd@hytc.edu.cn
}

Received 25 May 2019; Accepted 4 August 2019; Published 20 December 2019

Academic Editor: Allan C. Peterson

Copyright (c) 2019 Dandan Yang and Chuanzhi Bai. This is an open access article distributed under the Creative Commons Attribution License, which permits unrestricted use, distribution, and reproduction in any medium, provided the original work is properly cited.

\begin{abstract}
In this paper, we investigate the existence of solutions for a class of fractional boundary value problems with anti-periodic boundary value conditions with $\psi$-Caupto fractional derivative. By means of some standard fixed point theorems, sufficient conditions for the existence of solutions for the fractional differential inclusions with $\psi$-Caputo derivatives are presented. Our result generalizes the known special case if $\psi(x)=x$ and single known results to the multi-valued ones.
\end{abstract}

\section{Introduction}

Fractional calculus is a generalization of the ordinary differentiation and integration to arbitrary noninteger order $[1,2]$, which is a wonderful technique to understand of memory and hereditary properties of materials and processes. Some recent contributions to fractional differential equations have been carried out, see the monographs [3-6], and the references cited therein. Much attention has been focused on the study of anti-periodic boundary conditions, which are applied in different fields, such as blood flow problems, chemical engineering, underground water flow, populations dynamics, and so on, see the references ([7-9]) and paper cited therein. In 2009, Ahamad and Otero-Espinar [7] investigated the following fractional inclusions with anti-periodic boundary conditions

$$
\left\{\begin{array}{c}
{ }^{c} D^{q} x(t) \in F(t, x(t)), \quad t \in[0, T], \quad 1<q \leq 2, \\
x(0)=-x(T), \quad x^{\prime}(0)=-x^{\prime}(T) .
\end{array}\right.
$$

where ${ }^{c} D^{q} x(t)$ is the standard Caputo derivative of order $q, F:[0, T] \times R \rightarrow P(R)$ is a multivalued map, $\mathscr{P}(R)$ is the family of all subsets of $R$. Some sufficient conditions for the existence of solutions are given by means of BohnenblustKarlin fixed point theorem.

There are several definitions of fractional differential derivatives and integrals, such like Caputo type, RimannLiuville type, Hadamard type, and Erdelyi-Kober type and so on. In order to develop the fractional calculus, special kernels and some form of differential operator are chosen, see [10-16]. The $\psi$-Caputo fractional derivative of order $\alpha$, was first introduced by Almeida in [4]. Some properties, like semigroup law, Taylor's Theorem, Fermat's Thorem, etc., were presented. This new defined fractional derivative could model more accurately the process using differential kernels for the fractional operator.

In 2018, Samet and Aydi in [17] considered the following fractional differential boundary value problem with anti-periodic boundary conditions:

$$
\left\{\begin{array}{c}
{ }^{c} D^{\alpha, \psi} u(x)+f(x, u(x))=0, \quad a<x<b, \\
u(a)+u(b)=0, \quad u^{\prime}(a)+u^{\prime}(b)=0 .
\end{array}\right.
$$

where $\quad(a, b) \in R^{2}, a<b, \quad 1<\alpha<2, \quad \psi \in C^{2}([a, b])$, $\psi^{\prime}(x)>0, x \in[a, b]^{c} D^{\alpha, \psi}$ is the $\psi$-Caputo fractional derivative of order $\alpha$, and $f:[a, b] \times R \rightarrow R$ is a given function. A Lyapunov-type inequality is established. The authors also give some examples to illustrate the applications of their main results.

Inspired by the above works, we investigate the following anti-periodic fractional inclusions with $\psi$-Caputo derivatives:

$$
\left\{\begin{array}{l}
c D^{\alpha, \psi} u(x) \in F(x, u(x)), a<x<b, \\
u(a)+u(b)=0, \quad u^{\prime}(a)+u^{\prime}(b)=0 .
\end{array}\right.
$$

where $\quad(a, b) \in R^{2}, a<b, \quad 1<\alpha<2, \quad \psi \in C^{2}([a, b])$, $\psi^{\prime}(x)>0, x \in[a, b] .{ }^{c} D^{\alpha, \psi}$ is the $\psi$-Caputo fractional 
derivative of order $\alpha$, and $F:[a, b] \times R \rightarrow \mathscr{P}(R)$ is a multivalued map, $\mathscr{P}(R)$ is the family of all subsets of $R$. Sufficient conditions for the existence of solutions are given in view of the fixed point theorems for multi-valued mapping. The exposition in the framework of problem is new. If taking $a=0$, $b=T, \psi(x)=x$, the fractional differential inclusions (3) reduces to the fractional differential inclusions (1). If we take $F(x, u)=\{f(x, u)\}$, where $f:[a, b] \times R \rightarrow R$ is a given continuous function, then the problem (3) corresponds to the single-valued problem (2). The rest of this paper is organized as follows. We first present some basic definitions of fractional calculus, $\psi$-Caputo derivative and multi-valued maps. In Section 3, the main results on the existence of solutions for integral boundary value problem (3) are presented. An example is given to illustrate our main result in the last section.

\section{Preliminaries}

In this section, we recall some notations, definitions and preliminaries about fractional calculus [18-20], and $\psi$-Caputo fractional calculus $[4,17,21-23]$.

Definition 1 [9]. The Caputo fractional integral order $\alpha$ of a function $f \in C^{2}([0, T])$ is given by

$$
D_{0^{+}}^{\alpha} f(x)=\left(I_{0^{+}}^{2-\alpha} f^{\prime \prime}\right)(x), \quad 0<x<T,
$$

that is,

$$
D_{0^{+}}^{\alpha} f(x)=\frac{1}{\Gamma(2-\alpha)} \int_{0}^{x}(x-t)^{(1-\alpha)} f^{\prime \prime}(t) d t, \quad 0<x<T,
$$

Let $\psi \in C^{2}([0, T])$ be a given function such that

$$
\psi^{\prime}(x)>0,0 \leq x \leq T .
$$

Definition 2 [18]. The factional integral of order $\beta>0$ of a function $f \in C([0, T])$ with respect to $\psi$ is defined by

$$
\left(I_{0^{+}}^{\beta, \psi}\right) f(x)=\frac{1}{\Gamma(\beta)} \int_{0}^{x} \psi^{\prime}(t)(\psi(x)-\psi(t))^{\beta-1} f(t) d t, 0 \leq x \leq T .
$$

Definition 3 [4]. The $\psi$-Caputo fractional derivative of order $\alpha$ of a function $f \in C^{2}([0, T])$ is defined as

$$
\begin{aligned}
{ }^{C} D_{0^{+}}^{\alpha, \psi} f(x)= & \frac{1}{\Gamma(2-\alpha)} \int_{0}^{x} \psi^{\prime}(t)(\psi(x)-\psi(t))^{1-\alpha} \\
& \cdot\left(\frac{1}{\psi^{\prime}(t)} \frac{d}{d t}\right)^{2} f(t) d t, 0<x<T .
\end{aligned}
$$

Remark 1. Similarly, for $\psi \in C^{2}([a, b])$ and $\psi^{\prime}(x)>0$, $a \leq x \leq b$, the definition of $\psi$-Caputo fractional derivative of order $\alpha$ of a function $f \in C^{2}([a, b])$ could be given as follows:

$$
\begin{aligned}
{ }^{C} D_{\alpha^{+}}^{\alpha, \psi} f(x)= & \frac{1}{\Gamma(2-\alpha)} \int_{\alpha}^{x} \psi^{\prime}(t)(\psi(x)-\psi(t))^{1-\alpha} \\
& \cdot\left(\frac{1}{\psi^{\prime}(t)} \frac{d}{d t}\right)^{2} f(t) d t, \quad a<x<b .
\end{aligned}
$$

The following are definitions and properties concerning multi-valued maps $[9,24,25]$ which will be used in the remainder. A multivalued map $G: X \rightarrow \mathscr{P}(X)$ :

(i) Is called upper semicontinuous(u.s.c.) on $X$, if for each $x_{0} \in X$, the set $G\left(x_{0}\right)$ is a nonempty closed subset of $X$, and for each open set $N$ of $X$ containing $G\left(x_{0}\right)$, there exists an open neighborhood $N_{0}$ of $x_{0}$ such that $G\left(N_{0}\right) \subset N$.

(ii) The graph of $G$ is defined by the set $G r(G)=\{(x, y) \in X \times Y, y \in G(x)\}$.

(iii) $G$ is said to be measurable if for every $y \in R$, the function

$$
t \mapsto d(y, G(t))=\inf \{|y-z|: z \in G(t)\},
$$

is measurable.

(iv) If $G: X \rightarrow \mathscr{P}_{c l}(X)$ is called $\gamma$-Lipschitz if and only if there exists $\gamma>0$ such that

$$
H_{d}(N(x), N(y)) \leq \gamma d(x, y) \text {, for each } x, y \in X .
$$

(v) If $G: X \rightarrow \mathscr{P}_{c l}(X)$ is called contraction if and only if it is $\gamma$-Lipschitz with $\gamma<1$.

Let $Y$ be a separable metric space and let $N: Y \rightarrow \mathscr{P}\left(L^{1}(J, R)\right)$ be a multivalued operator. We call $N$ has a property (BC) if $N$ is lower semi-continuous (l.s.c.) and has nonempty closed and decomposable values. Let $F: J \times R \rightarrow \mathscr{P}(R)$ be a multivalued map with nonempty compact values. Define a multivalued operator $\mathscr{F}: C(J \times R) \rightarrow \mathscr{P}\left(L^{1}(J, R)\right)$ associated with $F$ as

$$
\mathscr{F}(x)=\left\{w \in L^{1}(J, R): w(t) \in F(t, x(t))\right\},
$$

for a.e. $t \in J$, which is called the Nemyskii operator associated with $F$. Let $F: J \times R \rightarrow \mathscr{P}(R)$ be a multivalued function with nonempty compact values. We say $F$ is of lower semi-continuous type (l.s.c. type) if its associated Nemytskii operator $\mathscr{F}$ is lower semi-continuous and has nonempty closed and decomposable values. Let $A$ be a subset of $J \times R$. A is $\mathscr{L} \times \mathscr{B}$ measurable if $A$ belongs to the $\sigma$-algebra generated by all sets of the form $\mathscr{J} \otimes \mathscr{D}$, where $\mathscr{J}$ is the Lebesgue measurable in $J$ and $\mathscr{D}$ is Borel measurable in $R$. A subset $\mathscr{A}$ of $L^{1}(J, R)$ is decomposable if for all $u, v \in \mathscr{A}$ and measurable $\mathscr{J} \subset J=[a, b]$, the function $u \mathscr{X}_{\mathscr{J}}+v \mathscr{X}_{J-\mathscr{J}} \in \mathscr{A}$, where $\mathscr{X}_{\mathscr{J}}$ stands for the characteristic function of $\mathscr{J}$. If the multi-valued map $G$ is completely continuous with nonempty compact values, then T is U.s.c. if and only if $G$ has a closed graph. For each $u \in C(J, R), J:=[a, b]$ is a closed interval from $a$ to $b$, denote the selection set of $F$ as

$$
S_{F, y}:=\left\{f \in L^{1}(J, R): f(t) \in F(t, u(t)) \text { a.e.t } \in J\right\} .
$$

Let $A, B \in \mathscr{P}_{c l}(X$.$) The Pompeiu-Hausdorff distance of A, B$ is defined by

$$
H_{d}(A, B)=\max \left\{\sup _{a \in A} d(a, B), \sup _{b \in B} d(A, b)\right\},
$$

where

$$
d(A, b)=\inf _{a \in A} d(a, b), d(a, B)=\inf _{b \in B} d(a, b)
$$


For convenience, we present the following notations.

$$
\begin{aligned}
P_{c l}(X) & =\{Y \in \mathscr{P}(X): Y \text { is closed }\}, P_{b}(X) \\
= & \{Y \in \mathscr{P}(X): Y \text { is bounded }\} . \\
P_{c p}(X)= & \{Y \in \mathscr{P}(X): Y \text { is compact }\}, P_{c p, c}(X) \\
= & \{Y \in \mathscr{P}(X): Y \text { is convex and compact }\} .
\end{aligned}
$$

To set the frame for our main results, we introduce the following lemmas.

Lemma 1 [26]. Let $(X, d)$ be a complete metric space. If $N: X \rightarrow \mathscr{P}_{c l}(X)$ is a contraction, then Fix $N \neq \varnothing$.

Lemma 2 [27]. (Nonlinear alternative for Kakutani maps). Let $E$ be a Banach space, $C$ a closed convex subset of $E, U$ an open subset of $C$ and $0 \in U$. Suppose that $F: \bar{U} \rightarrow \mathscr{P}_{c, c v}(C)$ is a upper semicontinuous compact map; here $\mathscr{P}_{c, c v}(C)$ denotes the family of nonempty, compact convex subsets of $C$. Then either

(i) F has a fixed point in $\bar{U}$, or

(ii) there is $a u \in \partial U$ and $\lambda \in(0,1)$ such that $u \in \lambda F(u)$.

Lemma 3 [28]. Let $X$ be a Banach space, and $F: J \times X \rightarrow(P)(X)$ be a $L^{1}$-Carathédory set-valued

map with $S_{F} \neq \varnothing$ and let $\Theta: L^{1}(J, X) \rightarrow C(J, X)$ be a linear continuous mapping. Then the set-valued map $\Gamma \circ S_{F}: C(J, X) \rightarrow \mathscr{P}(C(J, X))$ defined by

$$
\begin{gathered}
\left(\Theta \circ S_{F}\right)(u): C(J \times X) \rightarrow \mathscr{P}_{c p, c}(C(J, X)), \\
x \mapsto\left(\Theta \circ S_{F}\right)(u)=\Theta\left(S_{F, u}\right),
\end{gathered}
$$

is a closed graph operator in $C(J, X) \times C(J, X)$.

Lemma 4 [24]. Let $Y$ be a separable metric space and $N: Y \rightarrow \mathscr{P}\left(L^{1}(J, R)\right)$ be a multivalued operator satisfying the property (BC). Then $N$ has a continuous selection, that is, there exists a continuous function (single-valued) $g: Y \rightarrow L^{1}(J, R)$ such that $g(x) \in N(x)$ for every $x \in Y$.

Lemma 5 [29]. Let $h \in C([A, B]),(A, B) \in R^{2}, A<B$. Then $F \in C^{2}([A, B])$ is a solution to

$$
\begin{cases}\left({ }^{c} D_{A}^{\alpha} y\right)(t)=h(t), & t \in(A, B) \\ F(A)+F(B)=0, & F^{\prime}(A)+F^{\prime}(B)=0 .\end{cases}
$$

if and only if

$$
F(t)=\int_{A}^{B}(B-s)^{\alpha-2} H(t, s) h(s) d s, A \leq t \leq B,
$$

where

$$
H(t, s)= \begin{cases}\frac{1}{\Gamma(\alpha)}\left[\left(\frac{B-A}{4}-\frac{t-A}{2}\right)(\alpha-1)-\frac{B-s}{2}+\frac{(t-s)^{\alpha-1}}{(B-s)^{\alpha-2}}\right], & A \leq s \leq t<B, \\ \frac{1}{\Gamma(\alpha)}\left[\left(\frac{B-A}{4}-\frac{t-A}{2}\right)(\alpha-1)-\frac{B-s}{2}\right], & A \leq t \leq s<B .\end{cases}
$$

That is,

$$
\begin{aligned}
F(t)= & \frac{1}{\Gamma(\alpha)} \int_{A}^{B}\left[\left(\frac{B-A}{4}-\frac{t-A}{2}\right)(\alpha-1)(B-s)^{\alpha-2}\right. \\
& \left.-\frac{(B-s)^{\alpha-2}}{2}\right] h(s) d s+\frac{1}{\Gamma(\alpha)} \int_{A}^{t}(t-s)^{\alpha-1} h(s) d s .
\end{aligned}
$$

Lemma 6 [17]. If $f:[a, b] \times R \rightarrow R$, the problems

$$
\begin{cases}{ }^{c} D^{\alpha, \psi} u(x)=f(x, u(x)), & a<x<b \\ u(a)+u(b)=0, & u^{\prime}(a)+u(b)=0 .\end{cases}
$$

could be transformed into the following problems

$$
\begin{cases}\left({ }^{c} D_{\psi(a)}^{\alpha} v\right)(y)=f\left(\psi^{-1}(y), v(y)\right), & (a)<y<\psi(b), \\ v(\psi(a))+v(\psi(b))=0, & v^{\prime}(\psi(a))+v^{\prime}(\psi(b))=0,\end{cases}
$$

where $v \in C^{2}[A, B],(A, B)=(\psi(a), \psi(b))$. A nontrivial solution to (22) is given by

$$
v(y)=\int_{A}^{B}(B-s)^{\alpha-2} H(y, s) f\left(\psi^{-1}(s), v(s)\right) d s, A \leq y \leq B .
$$

i.e.,

$$
\begin{aligned}
v(y)= & \frac{1}{\Gamma(\alpha)} \int_{B}^{A}\left[\left(\frac{B-A}{4}-\frac{y-A}{2}\right)(\alpha-1)(B-s)^{\alpha-2}\right. \\
& \left.-\frac{(B-s)^{\alpha-1}}{2}\right] f\left(\psi^{-1}(s), v(s)\right) d s \\
& +\frac{1}{\Gamma(\alpha)} \int_{A}^{y}(y-s)^{\alpha-1} f\left(\psi^{-1}(s), v(s)\right) d s .
\end{aligned}
$$

From Lemma 6, we can easily know that

$$
\begin{aligned}
u(x)= & \frac{1}{\Gamma(\alpha)} \int_{a}^{b}\left[\left(\frac{\psi(b)-\psi(a)}{4}-\frac{\psi(x)-\psi(a)}{2}\right)\right. \\
& \left.\cdot(\alpha-1)(\psi(b)-\psi(t))^{\alpha-2}-\frac{(\psi(b)-\psi(t))^{\alpha-1}}{2}\right] \\
& \cdot \psi^{\prime}(t) f(t, u(t)) d t+\frac{1}{\Gamma(\alpha)} \int_{a}^{x}(\psi(x)-\psi(t))^{\alpha-1} \\
& \cdot \psi^{\prime}(t) f(t, u(t)) d t .
\end{aligned}
$$

\section{Main Results}

Now we are in the position to state our main results. 
3.1. The Lipschitz Case. $\left(A_{1}\right) F:[a, b] \times R \rightarrow \mathscr{P}_{c p}(R)$ is such that, for every $u \in R, F(\cdot, u)$ is measurable.

$\left(A_{2}\right)$ There exists $m \in L^{1}\left([a, b], R^{+}\right)$for almost all $t \in[a, b]$, such that

$$
d_{H}(F(t, u), F(t, \bar{u})) \leq m(t)|u-\bar{u}|, \forall u, \bar{u} \in R,
$$

with $d(0, F(t, 0)) \leq m(t)$ for almost all $t \in[a, b]$.

$\left(A_{3}\right) 1<\alpha<2, \psi \in C^{2}([a, b]), \psi^{\prime}(x)>0, x \in[a, b]$

$\left(A_{4}\right) \psi^{\prime}(a)=\psi^{\prime}(b)$.

Theorem 1. Suppose that $\left(A_{1}\right)-\left(A_{4}\right)$. If

$$
\begin{aligned}
& {\left[\frac{(\psi(b)-\psi(a))(\alpha-1)}{4 \Gamma(\alpha)} \int_{a}^{b}(\psi(b)-\psi(s))^{\alpha-2} \psi^{\prime}(s) m(s) d s\right.} \\
& \left.+\frac{3}{2 \Gamma(\alpha)} \int_{a}^{b}(\psi(b)-\psi(s))^{\alpha-1} \psi^{\prime}(s) m(s) d s\right]<1,
\end{aligned}
$$

then problem (3) has at least a solution in $[a, b]$.

Proof. By Lemma 6, we define the operator $T: C([a, b], R) \rightarrow \mathscr{P}(C[a, b], R)$ as follows:

$$
\begin{aligned}
T(u)= & \{h \in \mathrm{C}([a, b], R): h(t) \\
= & \frac{1}{\Gamma(\alpha)} \int_{a}^{b}\left[\left(\frac{\psi(b)-\psi(a)}{4}-\frac{\psi(t)-\psi(a)}{2}\right)\right. \\
& \left.\cdot(\alpha-1)(\psi(b)-\psi(s))^{\alpha-2}-\frac{(\psi(b)-\psi(s))^{\alpha-1}}{2}\right] \psi^{\prime}(s) f(s) d s \\
& \left.+\frac{1}{\Gamma(\alpha)} \int_{a}^{t}(\psi(t)-\psi(s))^{\alpha-1} \psi^{\prime}(s) f(s) d s, f \in S_{F, u}\right\} .
\end{aligned}
$$

We shall prove that the operator $T$ satisfies all the conditions in Lemma 1, thus $T$ has a fixed point that is a solution to the antiperiodic problem (3). First of all, for each $h \in C([a, b], R)$ the operator $T$ is closed. Let $\left\{h_{n}\right\}_{n \geq 0} \in T(u)$ be such that $h_{n} \rightarrow(n \rightarrow \infty)$ in $C([a, b], R)$. Then $h \in C([a, b], R)$, and there exists $v_{n} \in S_{F, u}$ such that for each $t \in[a, b]$,

$$
\begin{aligned}
h_{n}(t)= & \frac{1}{\Gamma(\alpha)} \int_{a}^{b}\left[\left(\frac{\psi(b)-\psi(a)}{4}-\frac{\psi(t)-\psi(a)}{2}\right)\right. \\
& \left.\cdot(\alpha-1)(\psi(b)-\psi(s))^{\alpha-2}-\frac{(\psi(b)-\psi(s))^{\alpha-1}}{2}\right] \\
& \cdot \psi^{\prime}(s) v_{n}(s) d s+\frac{1}{\Gamma(\alpha)} \int_{\alpha}^{t}(\psi(t))-(\psi(s))^{\alpha-1} \\
& \cdot \psi^{\prime}(s) v_{n}(s) d s .
\end{aligned}
$$

As $F$ has compact values, we pass onto a subsequence to get that $v_{n}$ converges to $v \in L^{1}([a, b],, R)$. Thus, $v \in S_{F, x}$, and for each $t \in[a, b]$, we have

$$
\begin{aligned}
h_{n}(t) \rightarrow h(t)= & \frac{1}{\Gamma(\alpha)} \int_{a}^{b}\left[\left(\frac{\psi(b)-\psi(a)}{4}-\frac{\psi(t)-\psi(a)}{2}\right)\right. \\
& \left.\cdot(\alpha-1)(\psi(b)-\psi(s))^{\alpha-2}-\frac{(\psi(b)-\psi(s))^{\alpha-1}}{2}\right] \\
& \cdot \psi^{\prime}(s) v(s) d s+\frac{1}{\Gamma(\alpha)} \int_{a}^{t}(\psi(t)-\psi(s))^{\alpha-1} \\
& \cdot \psi^{\prime}(s) v(s) d s .
\end{aligned}
$$

Thus, $h \in T(u)$.

Next, we will show there exists $\gamma<1$ such that

$$
H_{d}(F(t, u), F(t, \bar{u})) \leq \gamma\|u-\bar{u}\| .
$$

In fact, let $u, \bar{u} \in C([a, b], R)$ and $h_{1} \in T(u)$. There exists $v_{1}(t) \in F(t, u(t))$ such that for each $t \in[a, b]$,

$$
\begin{aligned}
h_{1}(t)= & \frac{1}{\Gamma(\alpha)} \int_{a}^{b}\left[\left(\frac{\psi(b)-\psi(a)}{4}-\frac{\psi(t)-\psi(a)}{2}\right)\right. \\
& \left.\cdot(\alpha-1)(\psi(b)-\psi(s))^{\alpha-2}-\frac{(\psi(b)-\psi(s))^{\alpha-1}}{2}\right] \\
& \cdot \psi^{\prime}(s) v_{1}(s) d s+\frac{1}{\Gamma(\alpha)} \int_{a}^{t}(\psi(t)-\psi(s))^{\alpha-1} \\
& \cdot \psi^{\prime}(s) v_{1}(s) d s .
\end{aligned}
$$

From $\left(A_{2}\right)$, we obtain

$$
d_{H}(F(t, u), F(t, \bar{u})) \leq m(t)|u-\bar{u}|, \forall u, \bar{u} \in R .
$$

Thus, there exists $w \in F(t, \bar{u}(t))$ such that

$$
\left|v_{1}(t)-w(t)\right| \leq m(t)|u(t)-\bar{u}(t)|, t \in[a, b] .
$$

Define $U:[a, b] \rightarrow \mathscr{P}(R)$ by

$$
U(t):=\left\{w \in R:\left|v_{1}(t)-w(t)\right| \leq m(t)|u(t)-\bar{u}(t)|\right\} .
$$

Since the multivalued operator $U(t) \cap F(t, \bar{u}(t))$ is measurable, there exists a function $v_{2}(t)$, which is a measurable selection for $U$. So $v_{2}(t) \in F(t, \bar{u}(t))$ and for each $t \in[a, b]$, we have

$$
\left|v_{1}(t)-v_{2}(t)\right| \leq m(t)|u(t)-\bar{u}(t)| .
$$

For each $t \in[a, b]$, define

$$
\begin{aligned}
h_{2}(t)= & \frac{1}{\Gamma(\alpha)} \int_{a}^{b}\left[\left(\frac{\psi(b)-\psi(a)}{4}-\frac{\psi(t)-\psi(a)}{2}\right)\right. \\
& \left.\cdot(\alpha-1)(\psi(b)-\psi(s))^{\alpha-2}-\frac{(\psi(b)-\psi(s))^{\alpha-1}}{2}\right] \\
& \cdot \psi^{\prime}(s) v_{2}(s) d s+\frac{1}{\Gamma(\alpha)} \int_{a}^{t}(\psi(t)-\psi(s))^{\alpha-1} \\
& \cdot \psi^{\prime}(s) v_{2}(s) d s,
\end{aligned}
$$

and one has 


$$
\begin{aligned}
\left|h_{1}(t)-h_{2}(t)\right| \leq & \frac{1}{\Gamma(\alpha)} \int_{a}^{b}\left[\left(\frac{\psi(b)-\psi(a)}{4}-\frac{\psi(t)-\psi(a)}{2}\right)\right. \\
& \left.\cdot(\alpha-1)(\psi(b)-\psi(s))^{\alpha-2}-\frac{(\psi(b)-\psi(s))^{\alpha-1}}{2}\right] \\
& \cdot \psi^{\prime}(s)\left|v_{1}(s)-v_{2}(s)\right| d s+\frac{1}{\Gamma(\alpha)} \int_{a}^{t}(\psi(t)-\psi(s))^{\alpha-1} \\
& \cdot \psi^{\prime}(s)\left|v_{1}(s)-v_{2}(s)\right| d s \\
\leq & \frac{(\psi(b)-\psi(a))(\alpha-1)}{4 \Gamma(\alpha)} \int_{a}^{b}(\psi(b)-\psi(s))^{\alpha-2} \\
& \cdot \psi^{\prime}(s)\left|v_{1}(s)-v_{2}(s)\right| d s \\
& +\frac{1}{2 \Gamma(\alpha)} \int_{a}^{b}(\psi(b)-\psi(s))^{\alpha-1} \psi^{\prime}(s)\left|v_{1}(s)-v_{2}(s)\right| d s \\
& +\frac{1}{\Gamma(\alpha)} \int_{a}^{b}(\psi(b)-\psi(s))^{\alpha-1} \psi^{\prime}(s)\left|v_{1}(s)-v_{2}(s)\right| d s \\
\leq & \frac{(\psi(b)-\psi(a))(\alpha-1)\|u-\bar{u}\|}{4 \Gamma(\alpha)} \\
+ & \frac{(\psi(b)-\psi(a))(\alpha-1)\|u-\bar{u}\|}{4 \Gamma(\alpha)} \\
& \cdot \int_{a}^{b}(\psi(b)-\psi(s))^{\alpha-2} \psi^{\prime}(s) m(s) d s \\
& +\frac{\|u-\bar{u}\|}{2 \Gamma(\alpha)} \int_{a}^{b}(\psi(b)-\psi(s))^{\alpha-1} \psi^{\prime}(s) m(s) d s \\
& +\frac{\|u-\bar{u}\|}{\Gamma(\alpha)} \int_{a}^{b}(\psi(b)-\psi(s))^{\alpha-1} \psi^{\prime}(s) m(s) d s \\
& \\
&
\end{aligned}
$$

Hence, we have

$$
\begin{aligned}
\left\|h_{1}-h_{2}\right\| \leq & {\left[\frac{(\psi(b)-\psi(a))(\alpha-1)}{4 \Gamma(\alpha)} \int_{a}^{b}(\psi(b)-\psi(s))^{\alpha-2} \psi^{\prime}(s) m(s) d s\right.} \\
& \left.+\frac{3}{2 \Gamma(\alpha)} \int_{a}^{b}(\psi(b)-\psi(s))^{\alpha-1} \psi^{\prime}(s) m(s) d s\right]\|u-\bar{u}\| .
\end{aligned}
$$

The same arguments discussed as (40), interchanging $u$ and $\bar{u}$ yields

$$
\begin{aligned}
H_{d} F(t, \bar{u}), F(t, u) \leq & {\left[\frac{(\psi(b)-\psi(a))(\alpha-1)}{4 \Gamma(\alpha)}\right.} \\
& \cdot \int_{a}^{b}(\psi(b)-\psi(s))^{\alpha-2} \psi^{\prime}(s) m(s) d s+\frac{3}{2 \Gamma(\alpha)} \\
& \left.\cdot \int_{a}^{b}(\psi(b)-\psi(s))^{\alpha-1} \psi^{\prime}(s) m(s) d s\right]\|\bar{u}-u\|, \\
\leq & \|\bar{u}-u\| .
\end{aligned}
$$

By (28), $T$ is a contraction. Thus, by Lemma 1 , we conclude that $T$ admits a fixed point which is a solution to problem (3). It completes the proof.

3.2. The Carathéodory Case. $\left(A_{5}\right) F:[a, b] \times R \rightarrow \mathscr{P}(R)$ is Carathéodory and has nonempty compact and convex values; $\left(A_{6}\right)$ there exist a continuous nondecreasing function $q:[0, \infty) \rightarrow[0, \infty)$ and a function $p \in C\left([a, b], R^{+}\right)$such that

$$
\begin{aligned}
& \|F(t, u)\|:=\sup \{|f|: f \in F(t, u)\} \leq p(t) q(\|u\|), \\
& \quad \text { for each }(t, x) \in[a, b] \times R .
\end{aligned}
$$

Theorem 2. Assume that $\left(A_{3}\right)-\left(A_{6}\right)$ hold. Moreover, if there exists a constant $M>0$, such that

$$
\frac{M}{q(M)\left[(\psi(b)-\psi(a))(\alpha-1) /(4 \Gamma(\alpha)) \int_{a}^{b}(\psi(b)-\psi(s))^{\alpha-2} \psi^{\prime}(s) p(s) d s+3 /(2 \Gamma(\alpha)) \int_{a}^{b}(\psi(b)-\psi(s))^{\alpha-2} \psi^{\prime}(s) p(s) d s\right]}<1
$$

Then the problem (3) has at least one solution on $[a, b]$.

Proof. Define the operator $T: C([a, b], R) \rightarrow \mathscr{P}(C[a, b], R)$ as follows:

$$
\begin{aligned}
T(u)= & \{h \in \mathrm{C}([a, b], R): h(t) \\
= & \frac{1}{\Gamma(\alpha)} \int_{a}^{b}\left[\left(\frac{\psi(b)-\psi(a)}{4}-\frac{\psi(t)-\psi(a)}{2}\right)\right. \\
& \left.\cdot(\alpha-1)(\psi(b)-\psi(s))^{\alpha-2}-\frac{(\psi(b)-\psi(s))^{\alpha-1}}{2}\right] \psi^{\prime}(s) f(s) d s \\
& \left.+\frac{1}{\Gamma(\alpha)} \int_{a}^{t}(\psi(t)-\psi(s))^{\alpha-1} \psi^{\prime}(s) f(s) d s, f \in S_{F, u}\right\} .
\end{aligned}
$$

We shall show that $T$ satisfies all the assumptions of Lemma 2. The proof is divided into 5 steps.
Step 1. $T$ is convex for each $x \in C([a, b], R)$. Since $S_{F, u}$ is convex, so it is obvious that this step is true.

Step 2. $T$ maps the bounded sets into bounded sets of $C([a, b], R)$. For a positive $r>0$, let $B_{r}=\{v \in C([a, b], R):\|v\| \leq r\}$ be a bounded ball in $C([A, B], R)$, then for $h \in T(u) \mathcal{u} \in B_{r}$, there exists $f \in S_{F, u}$ such that

$$
\begin{aligned}
h(t)= & \frac{1}{\Gamma(\alpha)} \int_{a}^{b}\left[\left(\frac{\psi(b)-\psi(a)}{4}-\frac{\psi(t)-\psi(a)}{2}\right)\right. \\
& \left.\cdot(\alpha-1)(\psi(b)-\psi(s))^{\alpha-2}-\frac{(\psi(b)-\psi(s))^{\alpha-1}}{2}\right] \\
& \cdot \psi^{\prime}(s) f(s) d s+\frac{1}{\Gamma(\alpha)} \int_{a}^{t}(\psi(t)-\psi(s))^{\alpha-1} \\
& \cdot \psi^{\prime}(s) f(s) d s .
\end{aligned}
$$

It follows that 


$$
\begin{aligned}
|h(t)| \leq & \frac{1}{\Gamma(\alpha)} \int_{a}^{b}\left[\left(\frac{\psi(b)-\psi(a)}{4}-\frac{\psi(t)-\psi(a)}{2}\right)\right. \\
& \left.\cdot(\alpha-1)(\psi(b)-\psi(s))^{\alpha-2}+\frac{(\psi(b)-\psi(s))^{\alpha-1}}{2}\right] \\
& \cdot \psi^{\prime}(s)|f(s)| d s+\frac{1}{\Gamma(\alpha)} \int_{a}^{x}(\psi(t)-\psi(s))^{\alpha-1} \psi^{\prime}(s)|f(s)| d s .
\end{aligned}
$$

By $\left(A_{6}\right)$, we obtain

$$
\begin{aligned}
\|h\| \leq & \frac{(\psi(b)-\psi(a))(\alpha-1) q(\|u\|)}{4 \Gamma(\alpha)} \int_{a}^{b}(\psi(b)-\psi(s))^{\alpha-2} \psi^{\prime}(s) p(s) d s \\
& +\frac{q(\|u\|)}{2 \Gamma(\alpha)} \int_{a}^{b}(\psi(b)-\psi(s))^{\alpha-1} \psi^{\prime}(s) p(s) d s \\
& +\frac{q(\|u\|)}{\Gamma(\alpha)} \int_{a}^{b}(\psi(b)-\psi(s))^{\alpha-1} \psi^{\prime}(s) p(s) d s \\
\leq & q(\|u\|)\left[\frac{(\psi(b)-\psi(a))(\alpha-1)}{4 \Gamma(\alpha)} \int_{a}^{b}(\psi(b)-\psi(s))^{\alpha-2} \psi^{\prime}(s) p(s) d s\right. \\
& \left.++\frac{3}{2 \Gamma(\alpha)} \int_{a}^{b}(\psi(b)-\psi(s))^{\alpha-1} \psi^{\prime}(s) p(s) d s\right], \\
\leq & q(r)\left[\frac{(\psi(b)-\psi(a))(\alpha-1)}{4 \Gamma(\alpha)} \int_{a}^{b}(\psi(b)-\psi(s))^{\alpha-2} \psi^{\prime}(s) p(s) d s\right. \\
& \left.+\frac{3}{2 \Gamma(\alpha)} \int_{a}^{b}(\psi(b)-\psi(s))^{\alpha-1} \psi^{\prime}(s) p(s) d s\right] .
\end{aligned}
$$

Step 3. $T$ maps bounded set into equicontinuous sets. Let $t_{1}, t_{2} \in[a, b]$, and $t_{1}<t_{2}, u \in B_{r}$, where $B_{r}$ is a bounded set in $C([a, b], R)$, for $u \in T(u)$, we have

$$
\begin{aligned}
\left|h\left(t_{2}\right)-h\left(t_{1}\right)\right| \leq & \frac{q(r)}{\Gamma(\alpha)} \int_{a}^{b} \frac{\psi\left(t_{2}\right)-\psi\left(t_{1}\right)}{2} \\
& \cdot(\alpha-1)(\psi(b)-\psi(s))^{\alpha-2} \psi^{\prime}(s) p(s) d s \\
& +\frac{q(r)}{\Gamma(\alpha)} \int_{\alpha}^{t_{1}}\left[\left(\psi\left(t_{2}\right)-\psi(s)\right)^{\alpha-1}-\left(\psi\left(t_{1}\right)-\psi(s)\right)^{\alpha-1}\right] \\
& \cdot \psi^{\prime}(s) p(s) d s \\
& +\frac{q(r)}{\Gamma(\alpha)} \int_{t_{1}}^{t_{2}}\left[\left(\psi\left(t_{2}\right)-\psi(s)\right)^{\alpha-1}-\left(\psi\left(t_{1}\right)-\psi(s)\right)^{\alpha-1}\right] \\
& \cdot \psi^{\prime}(s) p(s) d s,
\end{aligned}
$$

the right side hand of above inequality tends to 0 independent of $v \in B_{r}$ as $t_{1} \rightarrow t_{2}$. By means of Ascoli-Arzelá Theorem, $T$ is completely continuous.

Step 4. $T$ has a closed graph. Set $u_{n} \rightarrow u_{*}, h_{n} \in T\left(u_{n}\right)$ and $h_{n} \rightarrow h_{*}$. Then, we shall show that $h_{*} \in T\left(u_{*}\right)$. For $h_{n} \in T\left(u_{n}\right)$, there exist $f_{n} \in S_{F, u_{n}}$ such that

$$
\begin{aligned}
h_{n}(t)= & \frac{1}{\Gamma(\alpha)} \int_{a}^{b}\left[\left(\frac{\psi(b)-\psi(a)}{4}-\frac{\psi(t)-\psi(a)}{2}\right)\right. \\
& \left.\cdot(\alpha-1)(\psi(b)-\psi(s))^{\alpha-2}-\frac{(\psi(b)-\psi(s))^{\alpha-1}}{2}\right] \\
& \cdot \psi^{\prime}(s) f_{n}(s) d s+\frac{1}{\Gamma(\alpha)} \int_{a}^{t}(\psi(t)-\psi(s))^{\alpha-1} \\
& \cdot \psi^{\prime}(s) f_{n}(s) d s .
\end{aligned}
$$

Thus, it suffices to show that there exists $f_{*} \in S_{F, u_{*}}$, such that for each $t \in[a, b]$,

$$
\begin{aligned}
h_{*}(t)= & \frac{1}{\Gamma(\alpha)} \int_{a}^{b}\left[\left(\frac{\psi(b)-\psi(a)}{4}-\frac{\psi(t)-\psi(a)}{2}\right)\right. \\
& \left.\cdot(\alpha-1)(\psi(b)-\psi(s))^{\alpha-2}-\frac{(\psi(b)-\psi(s))^{\alpha-1}}{2}\right] \\
& \cdot \psi^{\prime}(s) f_{*}(s) d s+\frac{1}{\Gamma(\alpha)} \int_{a}^{t}(\psi(t)-\psi(s))^{\alpha-1} \psi^{\prime}(s) f_{*}(s) d s .
\end{aligned}
$$

Consider the continuous linear the operator $\Phi: L^{1}([a, b], R) \rightarrow C([a, b], R)$ as follows:

$$
\begin{aligned}
f \mapsto \Phi(f)(t)= & \frac{1}{\Gamma(\alpha)} \int_{a}^{b}\left[\left(\frac{\psi(b)-\psi(a)}{4}-\frac{\psi(t)-\psi(a)}{2}\right)\right. \\
& \left.\cdot(\alpha-1)(\psi(b)-\psi(s))^{\alpha-2}-\frac{(\psi(b)-\psi(s))^{\alpha-1}}{2}\right] \\
& \cdot \psi^{\prime}(s) f(s) d s+\frac{1}{\Gamma(\alpha)} \int_{a}^{t}(\psi(t)-\psi(s))^{\alpha-1} \\
& \cdot \psi^{\prime}(s) f(s) d s .
\end{aligned}
$$

Notice that $\left\|h_{n}-h\right\| \rightarrow 0$, as $n \rightarrow \infty$. Thus, by Lemma 2, $\Phi \circ S_{F}$ is a closed graph operator. Moreover, we have $h_{n}(t) \in \Phi\left(S_{F, u_{n}}\right)$. By $u_{n} \rightarrow u_{*}$, we get

$$
\begin{aligned}
h_{*}(t)= & \frac{1}{\Gamma(\alpha)} \int_{a}^{b}\left[\left(\frac{\psi(b)-\psi(a)}{4}-\frac{\psi(t)-\psi(a)}{2}\right)\right. \\
& \left.\cdot(\alpha-1)(\psi(b)-\psi(s))^{\alpha-2}-\frac{(\psi(b)-\psi(s))^{\alpha-1}}{2}\right] \\
& \cdot \psi^{\prime}(s) f_{*}(s) d s+\frac{1}{\Gamma(\alpha)} \int_{a}^{t}(\psi(t)-\psi(s))^{\alpha-1} \psi^{\prime}(s) f_{*}(s) d s .
\end{aligned}
$$

for some $f_{*} \in S_{F, u_{*}}$.

Step 5. We show that there exists a open set $U \subset C([a, b], R)$, with $u \notin T(u)$ for any $\eta \in(0,1)$ and all $u \in \partial U$. Let $\eta \in(0,1)$, $u \in \eta T(u)$. Then for $t \in[a, b]$, there exists $f \in S_{F, u}$ such that

$$
\begin{aligned}
h(t)= & \frac{1}{\Gamma(\alpha)} \int_{a}^{b}\left[\left(\frac{\psi(b)-\psi(a)}{4}-\frac{\psi(t)-\psi(a)}{2}\right)\right. \\
& \left.\cdot(\alpha-1)(\psi(b)-\psi(s))^{\alpha-2}-\frac{(\psi(b)-\psi(s))^{\alpha-1}}{2}\right] \\
& \cdot \psi^{\prime}(s) f(s) d s+\frac{1}{\Gamma(\alpha)} \int_{a}^{t}(\psi(t)-\psi(s))^{\alpha-1} \psi^{\prime}(s) f(s) d s .
\end{aligned}
$$

Similar to the discussion of Step 2, we have

$$
\begin{aligned}
\|h\| \leq & q(\|u\|)\left[\frac{(\psi(b)-\psi(a))(\alpha-1)}{4 \Gamma(\alpha)} \int_{a}^{b}(\psi(b)-\psi(s))^{\alpha-2} \psi^{\prime}(s) p(s) d s\right. \\
& \left.+\frac{3}{2 \Gamma(\alpha)} \int_{a}^{b}(\psi(b)-\psi(s))^{\alpha-1} \psi^{\prime}(s) p(s) d s\right] \\
\leq & q(M)\left[\frac{(\psi(b)-\psi(a))(\alpha-1)}{4 \Gamma(\alpha)} \int_{a}^{b}(\psi(b)-\psi(s))^{\alpha-2} \psi^{\prime}(s) p(s) d s\right. \\
& \left.+\frac{3}{2 \Gamma(\alpha)} \int_{a}^{b}(\psi(b)-\psi(s))^{\alpha-1} \psi^{\prime}(s) p(s) d s\right],
\end{aligned}
$$

which leads to 


$$
\frac{M}{q(M)\left[(\psi(b)-\psi(a))(\alpha-1) /(4 \Gamma(\alpha)) \int_{a}^{b}(\psi(b)-\psi(s))^{\alpha-2} \psi^{\prime}(s) p(s) d s+3 /(2 \Gamma(\alpha)) \int_{a}^{b}(\psi(b)-\psi(s))^{\alpha-1} \psi^{\prime}(s) p(s) d s\right]} \leq 1 .
$$

By (42), there exist $M$ such that $\|u\| \neq M$. Let

$$
U=\{x \in C([a, b], R):\|u\|<M\} .
$$

Note that the operator $T: \bar{U} \rightarrow \mathscr{P}(C([a, b], R))$ is upper semicontinuous and completely continuous. By the choice of $U$, there is no $x \in \partial U$ such that $u \in \eta T(u)$ for some $\eta \in(0,1)$. Thus, by means of Lemma 2 , we can get the conclusion that there exists a fixed point $u \in \bar{U}$, that is, it is a solution of problem (3). We complete the proof.

\subsection{The Lower Semicontinuous Case}

Theorem 3. Assume that $\left(A_{3}\right)-\left(A_{6}\right)$ and the following condition holds:

$\left(A_{7}\right) F:[a, b] \times R \rightarrow \mathscr{P}(R)$ is a nonempty compact-valued multivalued map such that

(a) $(t, u) \mapsto F(t, u)$ is $\mathscr{L} \otimes \mathscr{B}$ is measurable,

(b) $u \mapsto F(t, u)$ is lower semicontinuous for each $t \in[a, b]$, then the anti-periodic boundary problem (1.3) has at least one solution on $[a, b]$.

Proof. By $\left(A_{7}\right), F$ is of l.s.c. type. Then from Lemma 4, there exists a continuous function $f: C(J, R) \rightarrow L^{1}(J, R)$ such that $f(u) \in \mathscr{F}(u)$ for all $u \in C(J, R)$. Consider the following problem

$$
\begin{cases}\left(D_{\psi(a)}^{\alpha} u\right)(x)=f(u(x)), & \psi(a)<x<\psi(b) \\ u(\psi(a))+u(\psi(b))=0, & u^{\prime}(\psi(a))+u^{\prime}(\psi(b))=0\end{cases}
$$

If $u \in C^{2}([a, b], R)$ is a solution to (57), then $u$ is a solution to the problem (3). In order to transform the problem (57) into a fixed point problem, we define the operator $\overline{\mathscr{T}}$ as

$$
\begin{aligned}
\overline{\mathscr{T}} u(x)= & =\frac{1}{\Gamma(\alpha)} \int_{a}^{b}\left[\left(\frac{\psi(b)-\psi(a)}{4}-\frac{\psi(t)-\psi(a)}{2}\right)\right. \\
& \left.\cdot(\alpha-1)(\psi(b)-\psi(s))^{\alpha-2}-\frac{(\psi(b)-\psi(s))^{\alpha-1}}{2}\right] \\
& \cdot \psi^{\prime}(s) f(u(s)) d s+\frac{1}{\Gamma(\alpha)} \int_{a}^{t}(\psi(t)-\psi(s))^{\alpha-1} \\
& \cdot \psi^{\prime}(s) f(u(s)) d s .
\end{aligned}
$$

It is clear that $\overline{\mathscr{T}}$ is continuous and completely continuous. The remaining part of the proof is similar to that of Theorem 2, so we omit it here. The proof is complete.

Remark 4. If taking $a=0, . \quad b=T, \psi(x)=x$, the fractional differential inclusions (3) reduce to the fractional differential inclusions (1).

Remark 5. we take $F(x, u)=\{f(x, u)\}$, where $f:[a, b] \times R \rightarrow R$ is a given continuous function, then the problem (3) corresponds to the single-valued problem (2).

\section{Application}

Example 1. Consider the fractional differential inclusion involving $\psi$-Caputo derivative with anti-periodic boundary value conditions

$$
\left\{\begin{array}{l}
{ }^{c} D^{3 / 2, \psi} u(x) \in F(x, u(x)) \\
u(-1)+u(1)=0, u^{\prime}(-1)+u(1)=0
\end{array}\right.
$$

where $\psi(x)=\sinh (x),-1 \leq x \leq 1 . \alpha=3 / 2$. Obviously, condition $\left(A_{3}\right)$ is satisfied. Observe that $\psi \in C^{2}([-1,1])$, $\psi^{\prime}(x)=\cosh (x)>0,-1 \leq x \leq 1$. Moreover, we have

$$
\psi^{\prime}(-1)=\cosh (-1)=\cosh (1)=\psi^{\prime}(1) .
$$

Which implies condition $\left(A_{4}\right)$ holds.

$$
x \rightarrow F(x, u(x)):=\left[\frac{|u|^{5}}{|u|^{5}+3}+x^{2}+3, \frac{|u|}{|u|+1}+x^{3}+2\right], u \in R
$$

and

$$
\|F(x, u)\|:=\sup |v|: v \in F(x, u) \leq 5:=p(x) q(\|u\|) u \in R,
$$

where $p(x)=1, q(\|u\|)=5$, we can find a positive constant $M$ such that

$$
\frac{M}{5\left[(\sinh (1)-\sinh (-1)) / 4 \Gamma(3 / 2)(1 / 2) \int_{-1}^{1}(\sinh (1)-\sinh (s))^{(3 / 2)-2} \cosh (s) d s+3 /(2 \Gamma(3 / 2)) \int_{-1}^{1}(\sinh (1)-\sinh (s))(3 / 2)-2 \cosh (s) d s\right]}<1
$$

that is, $M>25.4125$. All the conditions in Theorem 2 are satisfied. Therefore, fractional differential inclusion with anti-periodic boundary value conditions (59) has at least one solution.

\section{Conflicts of Interest}

The authors declare that they have no conflicts of interest.

\section{Authors' Contributions}

The authors contributed equally to this paper. All authors read and approved the final manuscript.

\section{Acknowledgments}

This work is supported by Natural Science Foundation of China (11571136). 


\section{References}

[1] P. Agarwal, S. S. Dragomir, M. Jleli, and B. Samet, Advances in Mathematical Inequalities and Applications, Birkhauser, Singapore, 2018.

[2] M. Ruzhansky, Y. J. Cho, P. Agarwal, and I. Area, Advances in Real and Complex Analysis with Applications, Birkhauser, Singapore, 2017.

[3] B. Ahmad, A. Alsaedi, S. Aljoudi, and S. K. Ntouyas, "A sixpoint nonlocal boundary value problem of nonlinear coupled sequential fractional integro-differential equations and coupled integral boundary conditions," Journal of Applied Mathematics and Computing, vol. 56, no. 1-2, pp. 367-389, 2018.

[4] R. Almeida, "A caputo fractional derivative of a function with respect to another function," Communications in Nonlinear Science and Numerical Simulation, vol. 44, pp. 460-481, 2017.

[5] H. H. Alsulami, "Some existence results for differential inclusions of fractional order with nonlocal strip conditions," Advances in Difference Equations, vol. 2013, no. 1, Article ID 181, 10 pages, 2013.

[6] Y. Yue, Y. Tian, and Z. Bai, "Infinitely many nonnegative solutions for a fractional differential inclusion with oscillatory potential," Applied Mathematics Letter, vol. 88, pp. 64-72, 2019.

[7] B. Ahmad and V. Otero-Espinar, "Existence of solutions for fractional differential inclusions with antiperiodic boundary conditions," Boundary Value Problems, vol. 2009, Article ID 625347, pp. 1-11, 2009.

[8] B. Ahmad, S. K. Ntouyas, and A. Alsaedi, "On fractional differential inclusions with anti-periodic type integral boundary conditions," Boundary Value Problems, vol. 2013, no. 1, Article ID 82, 15 pages, 2013.

[9] K. Deimling, Multivalued Differential Equations, Walter De Gruyter, Berlin-NY, 1977.

[10] B. Ahmad and S. K. Ntouyas, "On Hadmard fractional integrodifferential boundary value problems," Journal of Applied Mathematics and Computing, vol. 47, no. 1-2, pp. 119-131, 2015.

[11] R. Almeida, A. B. Malinowska, and M. T. T. Monteiro, "Fractional differential equations with a caputo derivative with respect to a kernel function and their applications," Mathematical Methods in the Applied Sciences, vol. 41, no. 1, pp. 336-352, 2018.

[12] H. Baghani, "Existence and uniqueness of solutions to fractional langevin equations involving two fractional orders," Journal of Fixed Point Theory and Applications, vol. 20, no. 2, Article ID 63, 7 pages, 2018.

[13] J. V. da C. Sousa, F. G. Rodrigues, E. C. de Oliveira, "Stability of the fractional Volterra integro-differential equation by means of $\psi$-hilfer operator," Mathematical Methods in the Applied Sciences, vol. 42, pp. 3033-3043, 2019.

[14] D. Vivek, K. Kanagarajan, and E. M. Elsayed, "Some existence and stability results for hilfer-fractional implicit differential equations with nonlocal conditions," Mediterranean Journal of Mathematics, vol. 15, no. 1, 2018.

[15] George Z. Voyiadjis and W. Sumelka, "Brain modelling in the framework of anisotropic hyperelasticity with time fractional damage evolution governed by the Caputo-Almeida fractional derivative," Journal of the Mechanical Behavior of Biomedical Materials, vol. 89, pp. 209-216, 2019.

[16] X.-J. Yang and J. A. Tenreiro Machado, "A new fractional operator of variable order: application in the description of anomalous diffusion," Physica A: Statistical Mechanics and its Applications, vol. 481, pp. 276-283, 2017.

[17] B. Samet and H. Aydi, "Lyapunov-type inequalities for an antiperiodic fractional boundary value problem involving $\psi$-Caputo fractional derivative," Journal of Inequalities and Applications, vol. 2018, no. 1, Article ID 286, 11 pages, 2018.

[18] A. A. Kilbas, H. M. Srivastava, and J. J. Trujillo, "Theory and applications of fractional differential equations," North-Holl and Mathematics Studies, vol. 204, Elsevier, Amsterdam, 2006.

[19] V. Lakshmikantham and A. S. Vatsala, "Basic theory of fractional differential equations," Nonlinear Analysis: Theory, Methods \& Applications, vol. 69, no. 8, pp. 2677-2682, 2008.

[20] S. G. Samko, A. A. Kilbas, and O. I. Marichev, Fractional Integrals and Derivatives: Theory and Applications, Gordon and Breach, Yverdon, 1993.

[21] P. Agarwal, S. Jain, and T. Mansour, "Further extended caputo fractional derivative operator and its applications," Russian Journal of Mathematical Physics, vol. 24, no. 4, pp. 415-425, 2017.

[22] M. Awadalla and Y. Y. Yameni, "Modeling exponential growth and exponential decay real phenomena by $\psi$-Caputo fractional derivative," Journal of Advances in Mathematics and Computer Science, vol. 28, no. 2, pp. 1-13, 2018.

[23] I. O. Kiymaz, A. Cetinkaya, and P. Agarwal, "An extension of caputo fractional derivative operator and its applications," Journal of Nonlinear Sciences and Applications, vol. 9, no. 6, pp. 3611-3621, 2016.

[24] A. Bressan and G. Colombo, "Extensions and selections of maps with decomposable values," Studia Mathematica, vol. 90, no. 1, pp. 69-86, 1988.

[25] S. N. Papageorgiou, Handbook of Multivalued Analysis. Theory I, Kluwer, Dordrecht, 1997.

[26] H. Covitz and S. B. Nadler, "Multi-valued contraction mappings in generalized metric spaces," Israel Journal of Mathematics, vol. 8, no. 1, pp. 5-11, 1970.

[27] A. Granas and J. Dugundji, Fixed Point Theory, Springer-Verlag, NY, 2005.

[28] A. Lasota and Z. Opial, "An application of the Kakutani-Ky Fan theorem in the theory of ordinary differential equations," Bulletin of Polish Academy of Sciences, Science Series Mathematics, Astronomy and Physics, vol. 13, pp. 781-786, 1965.

[29] R. A. C. Ferreira, "Lyapunov-type inequality for an anti-periodic fractional boundary value problem," Fractional Calculus and Applied Analysis, vol. 20, no. 1, pp. 284-291, 2017. 


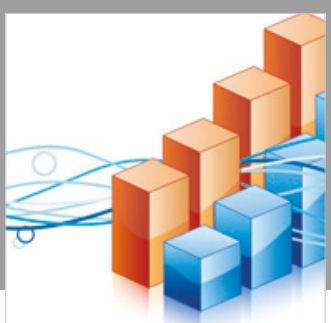

Advances in

Operations Research

\section{-n-m}
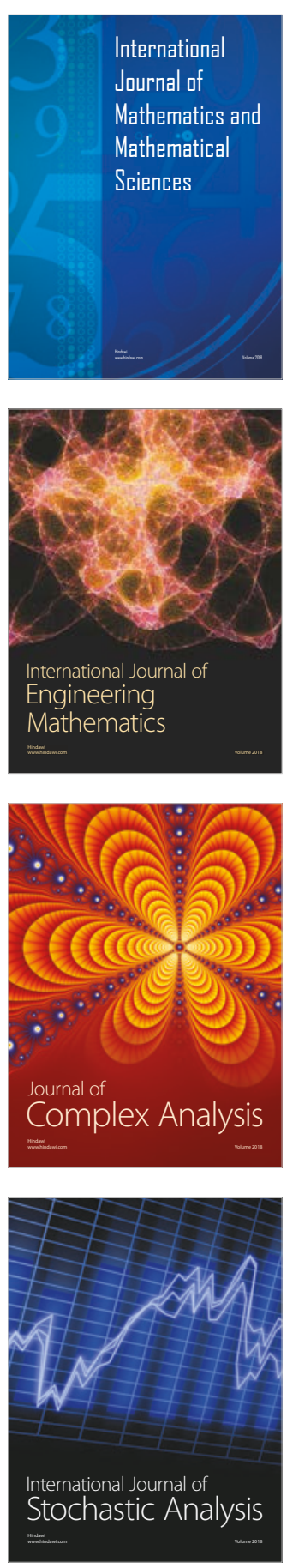
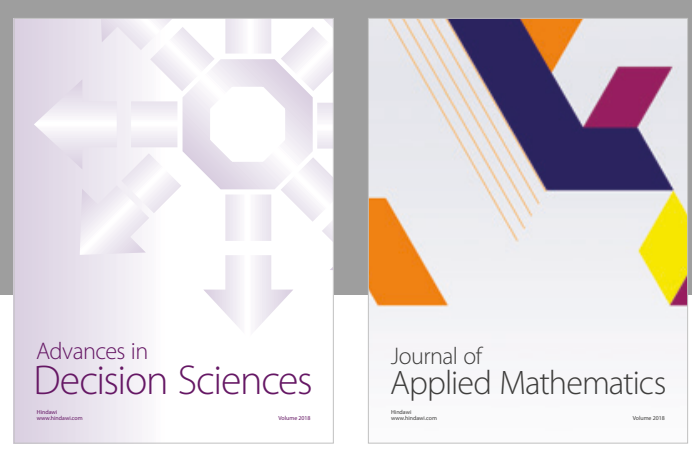

Journal of

Applied Mathematics
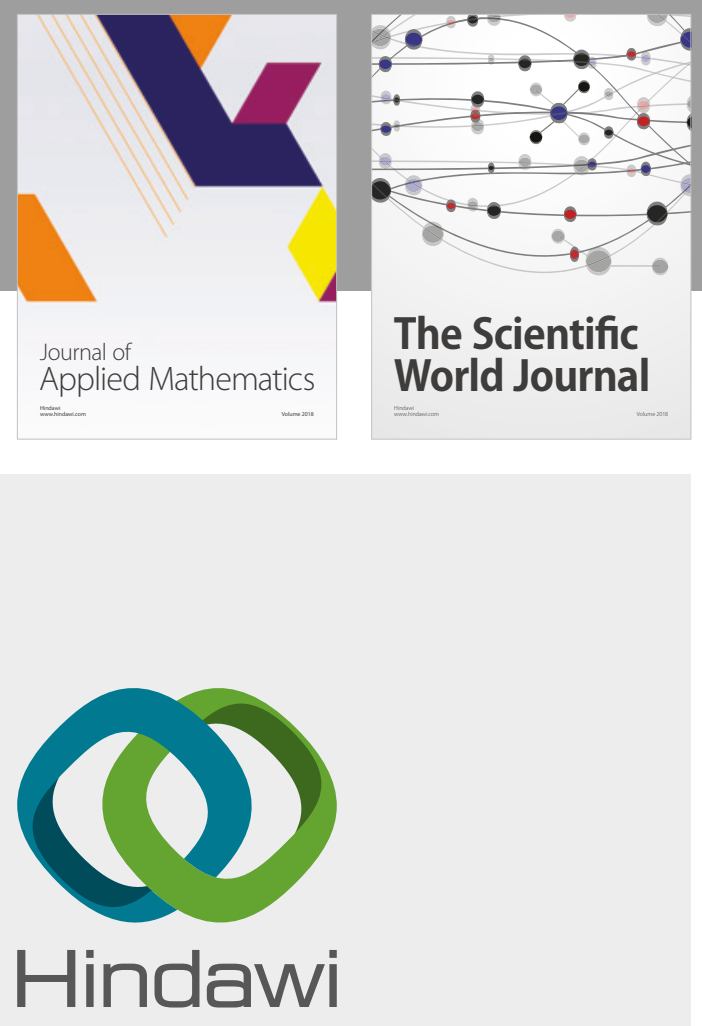

Submit your manuscripts at

www.hindawi.com

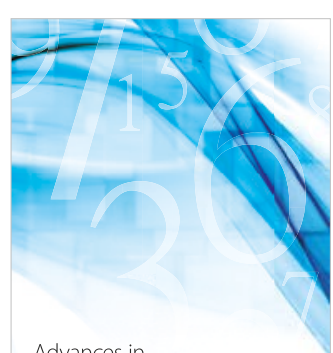

Advances in
Numerical Analysis
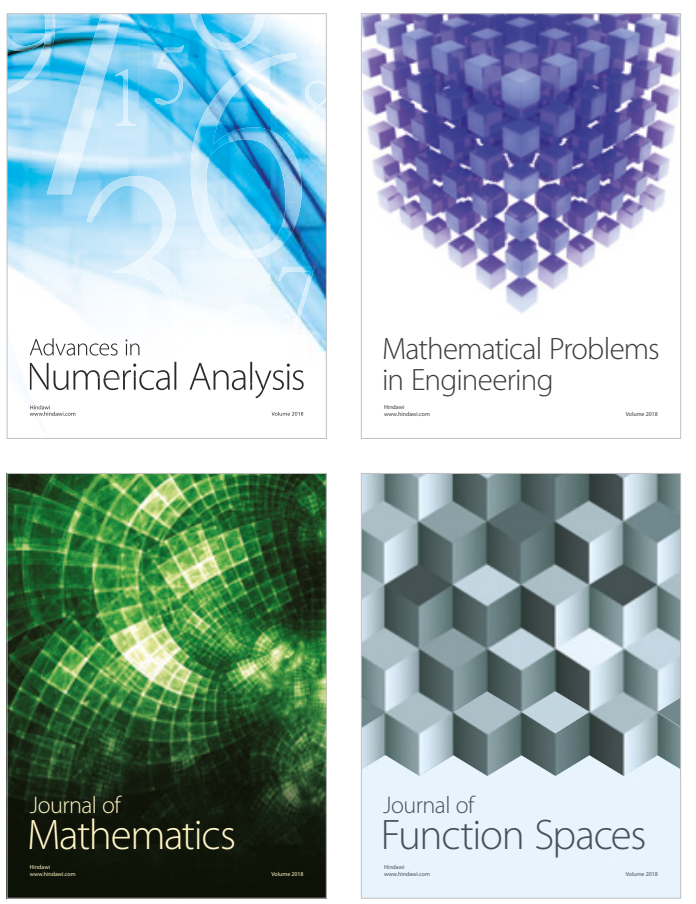

Mathematical Problems in Engineering

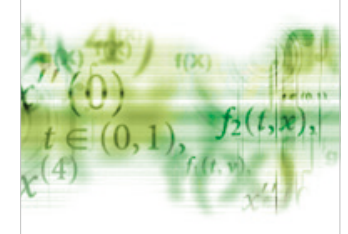

International Journal of

Differential Equations

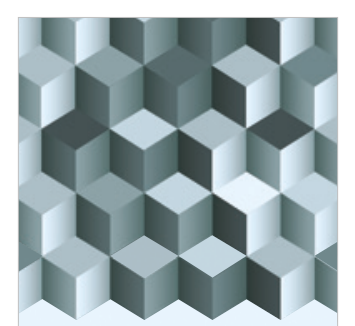

Journal of

Function Spaces
The Scientific

World Journal

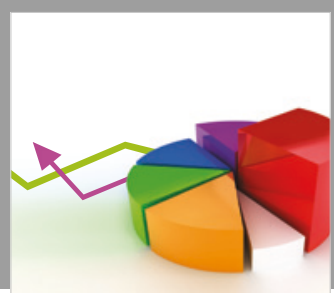

Journal of

Probability and Statistics
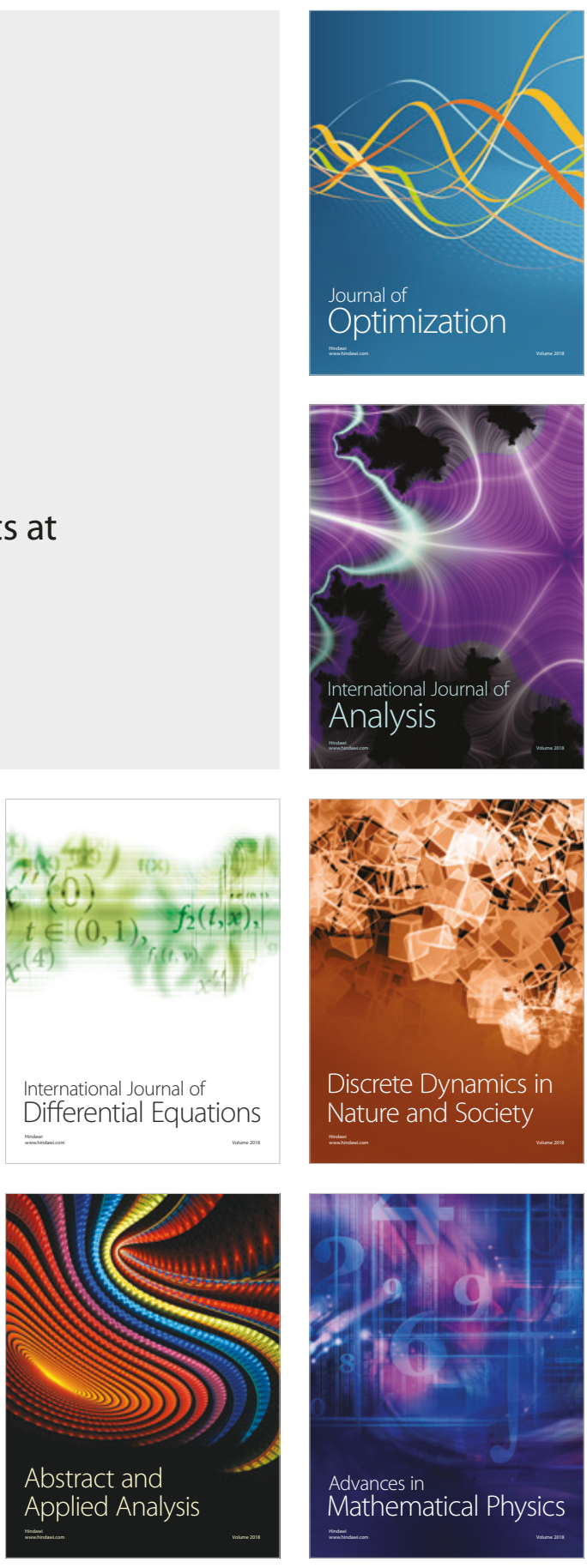